\title{
Computer Simulation of Cyclic Polymers in Disordered Media
}

\author{
Aleksander Kuriata, Andrzej Sikorski* \\ Department of Chemistry, University of Warsaw \\ Pasteura 1, 02-093 Warsaw, Poland \\ *E-mail: sikorski@chem.uw.eu.pl \\ Phone: +4822822 0211, Fax: +48228225996
}

Received: 21 November 2014; revised: 26 February 2015; accepted: 27 February 2015; published online: 18 March 2015

\begin{abstract}
In order to determine the structure and dynamical properties of cyclic polymers (rings) in a random environment we developed and studied an idealized model. All atomic details were suppressed, chains were represented as a sequence of identical beads and were embedded to a simple cubic lattice. A set of obstacles was also randomly introduced into the system and it can be viewed as a model of porous media. A Monte Carlo sampling algorithm using local changes of chain conformation was used to sample the conformational space. It was shown that the mean dimensions of the chain changed with the concentration of obstacles but these changes were non-monotonic. The long-time (diffusion) dynamic properties of the system were also studied. The differences in the mobility of chains depending on the obstacle density were shown and discussed.
\end{abstract}

Key words: cyclic polymers, lattice models, Monte Carlo method, disordered media

\section{INTRODUCTION}

The structure and dynamic properties of polymer chains in a random environment like porous media is an interesting and challenging problem and it has recently been a subject of many experimental and theoretical studies [1-2]. This is because of the practical importance of coating the surfaces, laminates, chromatography, colloidal stabilization etc. [3]. Simple lattice models of linear and non-linear (cyclic, star-branched) polymer chains confined in a slit were a subject of many computer simulation studies [4]. The introduction of obstacles into the slit containing a polymer chain was treated as a crude model of macromolecules in porous media (quenched disorder) [1]. The scaling properties of chains, changes of their shape and density distributions were determined for such systems [1]. Theoretical considerations and computer simulations concerning macromolecules in a system containing impenetrable obstacles (rods, spheres) in three- and two-dimensional space were also frequently performed [5-20].

Cyclic chains are also of great interest especially due to their different dynamic properties in dense melts when compared to linear and branched macromolecules [21-23]. Rings were studied in real experiments [23-28], theoretically [2931 ] and by means of computer simulation [32-44]. It was shown that the scaling behavior of single (isolated) cyclic polymers was very similar to linear ones. However, size of the rings (squared radius of gyration $S^{2}$ ) in the melt scaled with their length as $N^{0.83}$ instead of $N^{1}$ while the dynamics was similar: the self-diffusion coefficient $D \sim N^{-1.59}$ [41, 43]. Moreover, very long cyclic chains in melt are collapsed and the scaling of their size was as $N^{2 / 3}[42,44]$. The formation of catenated structures and its influence on the properties 
of the macromolecular system was also studied [45]. Cyclic chains in the system of obstacles (a model of disordered media) were studied by computer simulations [46-48] and theoretical deliberations [29-30]. The behavior of rings in disordered media was predicted to be different than those of linear and star-branched chains: long macromolecules consisted of $N$ elements, the squared radius of gyration scaled as $N^{1}$ while the self-diffusion coefficient as $N^{-2}$.

In this paper we studied the influence of small obstacles on the structure and dynamic properties of a single cyclic polymer chains. For this purpose we used a coarse grained model of chains embedded in a simple cubic lattice. This lattice approximation should not affect the results as we are not interested in the local structure but we studied parameters describing the chain as a whole. The polymers were modeled at good solvent conditions and the excluded volume was the only polymer-polymer potential that was used. Properties of the model polymer system were determined by means of the Monte Carlo simulations with the sampling algorithm of a Verdier-Stockmayer type using local modifications of chain conformation.

\section{THE MODEL AND THE SIMULATION ALGORITHM}

The coarse-grained model of macromolecules was realized by the construction of cyclic sequences of $N$ identical beads, where a single bead represented some chemical mers. This idealized model was found to be sufficient for the studies of properties of a chain as a whole. In order to make the calculations more efficient we also introduced a lattice approximation: the positions of these polymer beads in space were limited to vertices of a simple cubic lattice. The excluded volume was the only potential introduced into the model and the chains could not cross themselves. Other long-distance interactions were assumed to be zero, the system was athermal and the polymer chains were studied at good solvent conditions. Obstacles that were impenetrable for polymer beads were randomly introduced into the system. The size of an obstacle was the same as that of a polymer bead. The excluded volume was the only potential between obstacles and polymers. The model system consisting of one cyclic macromolecule and $\mathrm{m}$ obstacles was put into a large Monte Carlo box - the edge of the box was chosen to be large enough when compared to the chain's diameter in order to avoid the chain interacting with its image: $L \gg 2\left\langle S^{2}\right\rangle$.

The properties of the systems under consideration were determined by means of a computer simulation employing a Monte Carlo algorithm based on local changes of chain conformation. The set of these local moves consisted of: 1-bead motion, 2-bead motion and 2-bead crankshaft motion [49]. A polymer bead was picked at random and an attempt of a local motion was performed and accepted/rejected due to geometrical constraints. The above set of micromodification was found insufficient for dense polymer systems. Usually, the possibility of reptation was introduced into the model in such a case, but cyclic chains do not have ends and thus we have to use a different move. Thus, we decided to introduce wave motions where one bond is replaced by a sequence of three bonds forming U-shape. This modification of the chain conformation is not local as it shifts a fragment of chain along the chain contour for distances comparable with the number of beads [50]. All types of elementary moves were employed with the same frequency because such a procedure was efficient and provided the correct time scale in the simulation $[49,51]$. The time unit was defined as one attempt of each type of local motions per polymer bead on average.

An initial polymer conformation was constructed in a process of the simultaneous growing and equilibration procedure starting from the shortest possible chain, i.e. the square consisting of $N=4$ beads [49]. Then, the obstacles were added randomly. For each system under consideration 30-40 independent Monte Carlo simulation runs were performed for a different set of obstacles. Each Monte Carlo run consisted of $10^{9}-10^{10}$ time units and it started from a different initial configuration of chains and different sets of obstacles. At the start of the each simulation run the equilibration run was performed, which lasted $10^{7}-10^{8}$ time units. The criterion of the system equilibration was the stability of some parameters of the system, such as radius of gyration and the center-of-mass autocorrelation function.

\section{RESULTS AND DISCUSSION}

The simulations were performed for cyclic chains consisting of $N=50,100,200,400$ and 800 . This range of chain lengths allows studying the long-time behavior of such systems, i.e. the self-diffusion of macromolecules. These lengths are also sufficient to avoid the influence of lattice approximation. The density of obstacles $\rho_{\text {obs }}$ was defined as a fraction of sites in the Monte Carlo box occupied by these impenetrable objects, i.e.:

$$
\rho_{o b s}=\frac{m l^{3}}{L^{3}},
$$

where $\mathrm{m}$ is the number of obstacles, $L$ is the size of the Monte Carlo box and $l=1$ is the lattice unit. The density of obstacles was changed between $\rho_{o b s}=0$ and 0.25 , i.e. in the range where the sampling algorithm is still efficient. The size of the box was chosen $L=100$ lattice units.

The size of a model chain is usually described by means of the mean-squared radius of gyration $\left\langle S^{2}\right\rangle$ calculated as

$$
\left\langle S^{2}\right\rangle=\frac{1}{N} \sum_{i=1}^{N}\left\langle\left(r_{i}-r_{\mathrm{cm}}\right)^{2}\right\rangle,
$$


where $r_{i}$ is a vector denoting a position of a $i$-th bead and $r_{\mathrm{cm}}$ denotes the position of the chain's center-of-mass position. Fig. 1 presents the mean-squared radius of gyration $\left\langle S^{2}\right\rangle$ as a function of the chain length $N$ for three different obstacle densities. One can observe that the size of the chain is not affected by the presence of a small number of obstacles $\left(\rho_{o b s}=0.1\right)$. Introduction of the further amount of obstacles leads eventually to the chain contraction.

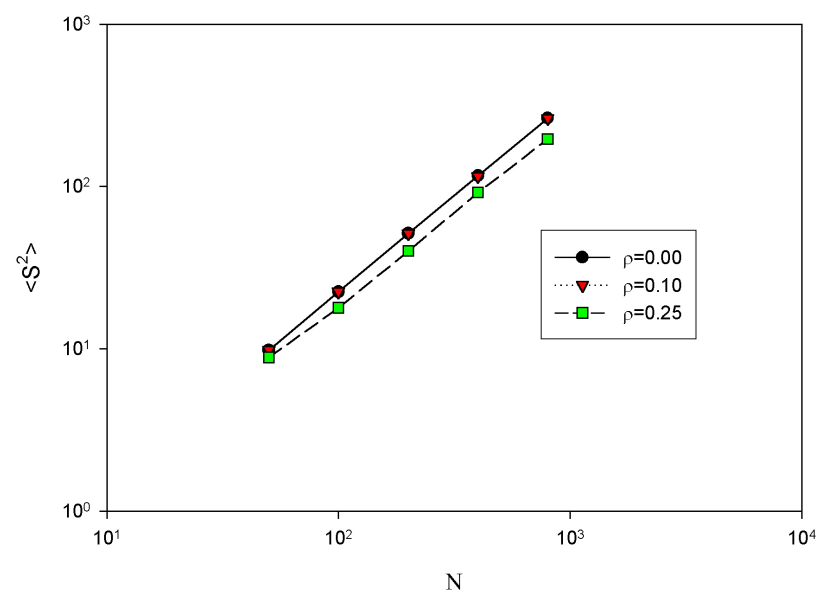

Fig. 1. The mean-squared radius of gyration $\left\langle S^{2}\right\rangle$ of the ring polymer as a function of the chain length $N$. The densities of obstacles $\rho_{\text {obs }}$ are given in the inset

In all cases the size of the chain scales with its length like $N^{\gamma}$, and the scaling exponent was found $\gamma=1.187 \pm 0.002$ for $\rho_{o b s}=0$ (a chain without obstacles), $1.185 \pm 0.003$ for $\rho_{o b s}=0.1$ and $1.155 \pm 0.009$ for $\rho_{o b s}=0.25$, respectively. Other simulation models (long chains on face-centered cubic lattice) estimated the exponent as 1.186 [37]. Recent experimental studies concerning polystyrenes in benzene-d6 gave this exponent $1.20 \pm 0.04$ [52] while theoretical predictions varied between 1.11 and 1.176 [53-54]. The decrease of the scaling exponent with the increase of the density of obstacles was rather small when compared to the behavior a cyclic polymers in melt where $S^{2} \sim N^{0.83}$ [42] On the other hand, it was considerably higher than that predicted theoretically from scaling considerations for rings in gel, where $S^{2} \sim N^{1}$ [29-30]. This difference can be explained by the fact that gel contains chemical crosslinks and topological entanglements that significantly reduce available conformational space. Moreover, small obstacles introduced to our model system are apparently at lower densities than polymer gel and below the percolation threshold (this threshold is 0.3116 for a simple cubic lattice).

The long-time dynamic properties of the polymer are usually studied by the analysis of the mean-squared displacement. We studied the center-of-mass autocorrelation function $g_{\mathrm{cm}}(t)$ defined as:

$$
g_{\mathrm{cm}}(t)=\left\langle\left[r_{\mathrm{cm}}(t)-r_{\mathrm{cm}}(t=0)\right]^{2}\right\rangle
$$

where $r_{\mathrm{cm}}$ stands for the center-of-mass position. This function is more statistically stable than the mean-squared displacement. In normal (Fickian) diffusion the mean-squared displacement scales as $t^{1}$ but in disordered systems the anomalous diffusion appears, i.e. $g_{\mathrm{cm}}(t)$ scales as $t^{a}$ with $a<1$. This relation is valid below the percolation threshold and the exponent for longer times approaches the value 1 again [6]. Fig. 2 presents the autocorrelation function for the chain $N=50$ for various densities of obstacles. One can observe that the upper curve (no obstacles) scales a $t^{1}$ in the entire ranges of time. Introduction of the small number of obstacles $\left(\rho_{o b s}=0.01\right.$ and 0.05$)$ does not change the scaling behavior or the region with the subdiffusion is very narrow. For higher obstacles densities $\left(\rho_{o b s}=0.05\right)$ one can observe regions with the exponent $a<1$. This subdiffusive behavior is apparently caused by caging the chains and thus hindering them.

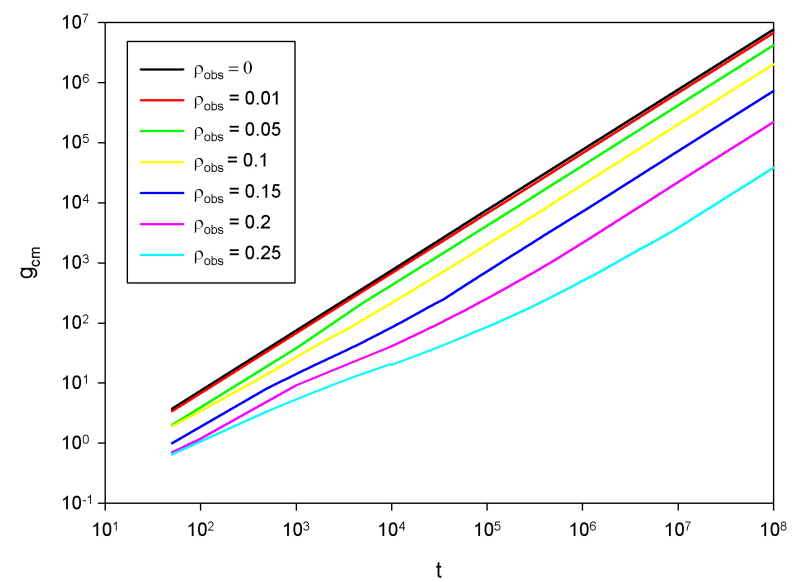

Fig. 2. The center-of-mass autocorrelation function for the chain $N=50$. The densities of obstacles $\rho_{o b s}$ are given in the inset

The diffusion coefficient $D$ was calculated according to the Einstein relation:

$$
D=\frac{g_{\mathrm{cm}}(t)}{6 t}
$$

where $t$ is time. The diffusion coefficient was determined for longer displacements of the chain where the $g_{\mathrm{cm}}$ function scales as $t^{1}$ [55]. In Fig. 3 the diffusion coefficient $D$ was plotted against the chain length $N$. One can observe the scaling behavior in all obstacle densities. The diffusion coefficient scales as $N^{-1.00 \pm 0.01}, N^{-1.21 \pm 0.03}, N^{-1.25 \pm 0.07}$ for the density $\rho_{o b s}=0,0.1$ and 0.25 , respectively. The same influence of the chain length and the density of the concentration was found experimentally for circular DNA [28]. In a cyclic polymer melt the scaling of the diffusion coefficient was found $D \sim N^{-1.59}$ [41] while theoretical predictions for a ring in gel (obstacles) gave stronger dependency: $D \sim N^{-2}$ [29-30]. 


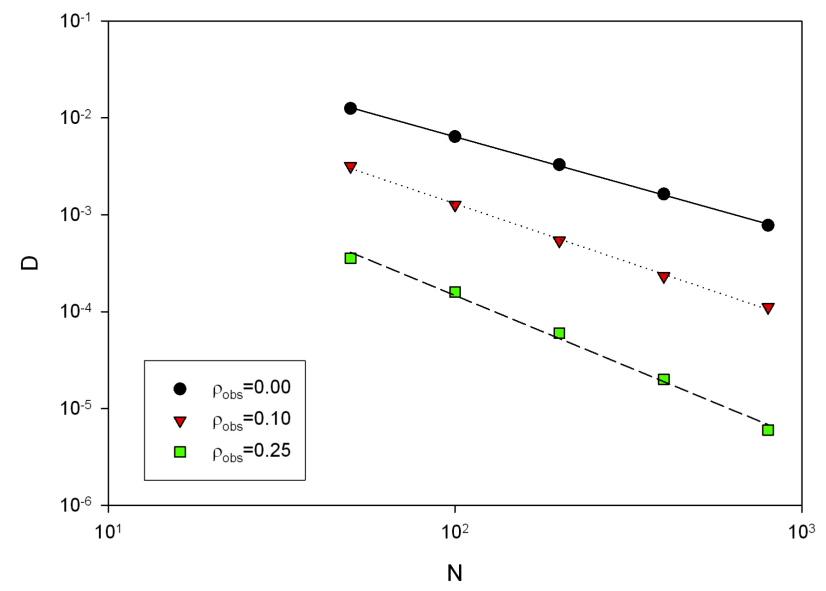

Fig. 3. The polymer self-diffusion coefficient $D$ of the ring polymer as a function of the chain length $N$. The densities of obstacles $\rho_{o b s}$ are given in the inset

Besides the above scaling behavior of size and dynamic parameters we studied the influence of the obstacles density on the chain properties. In Fig. 4 we present the reduced mean-squared radius of gyration $\left\langle S^{2}\right\rangle / N^{1.2}$ as a function of the obstacles density $\rho_{\text {obs }}$ for different lengths (this parameter was divided by $N^{1.2}$ in order to make the results obtained for all chains under consideration comparable. Exponent 1.2 was chosen because the size of a free chain scales as $N^{1.2}$.

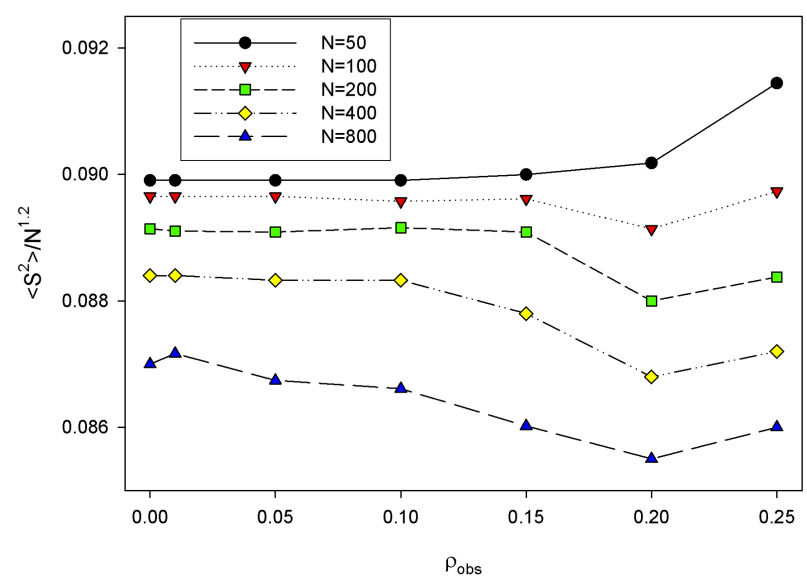

Fig. 4. The reduced mean-squared radius of gyration $\left\langle S^{2}\right\rangle / N^{1.2}$ as a function of the density of obstacles $\rho_{\text {obs }}$. The chain lengths are given in the inset

One can observe that in a wide range of obstacle densities the size of chains is almost constant $\left(\rho_{o b s}<0.15\right)$. Further increase of the obstacle density led to the increase of the chain size. This behavior was rather expected as recently theoretical deliberations (PRISM/polymer reference interaction site model theory) and computer simulations of linear chains in a similar environment revealed non-monotonic dependency of the radius of gyration on the density of obsta- cles: the size of the linear chain decreased for very low obstacle densities and increased moderately (PRISM theory) or rapidly (Monte Carlo simulations): the size of linear chains was stable for low densities of obstacles and above density 0.05 the size of the polymer increased [19]. The only differences in the behavior of linear and cyclic chains in random media were that the increase for rings began at higher densities, i.e. for $\rho_{o b s}>0.15$. The increase of the size of cyclic chains is smaller than the linear ones. This can be explained by the fact that linear polymers have free ends and thus they can much easier expand and their expansion is mainly originated from the ends. The expansion of rings can be realized by cooperative extension of parts of chains but this motion is considerably hindered because of constraints. The behavior of linear, branched and branched chains was found different in systems containing larger obstacles like rods where the chain size decreased for obstacle densities [4].

Fig. 5 presents the dependency of the diffusion coefficient $D$ on the density of obstacles $\rho_{\text {obs }}$ for all chain lengths under consideration. One can observe that the decrease of the chain mobility with the increase of the obstacle density is similar for all chains. Two regimes can be distinguished in the behavior of the self-diffusion coefficient. At lower densities of obstacles the dependence is exponential, i.e. $D$ $\sim \exp \left(-a \rho_{o b s}\right)$ and a is roughly equal to 2 . For the density of obstacles above 0.2 the decrease of the chain mobility becomes more rapid but it is impossible to clearly identify the scaling behavior as the simulation algorithm becomes inefficient.

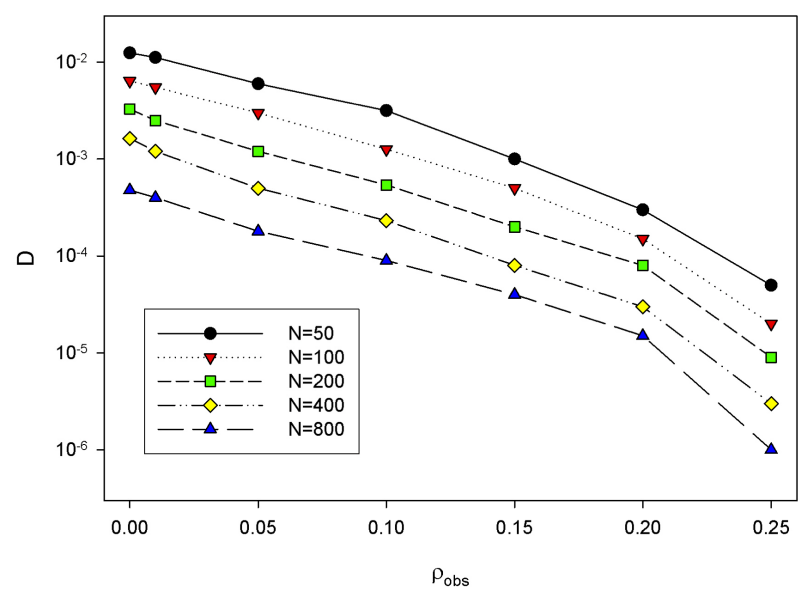

Fig. 5. The polymer self-diffusion coefficient $D$ as a function of the density of obstacles $\rho_{\text {obs }}$. The chain lengths are given in the inset

Visualization of the studied systems is presented in Fig. 6. Typical conformations of a short chain $(N=50)$ at low and high density of obstacles look similar although we know that for higher obstacle density the size of the macromolecule is higher for short chains (see Figure 3 and the discussion above). The chain at higher obstacle density has more extended fragments (trans conformations) but, on 
(a)

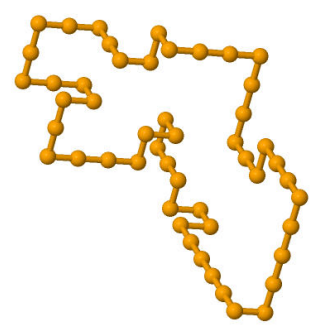

(c)

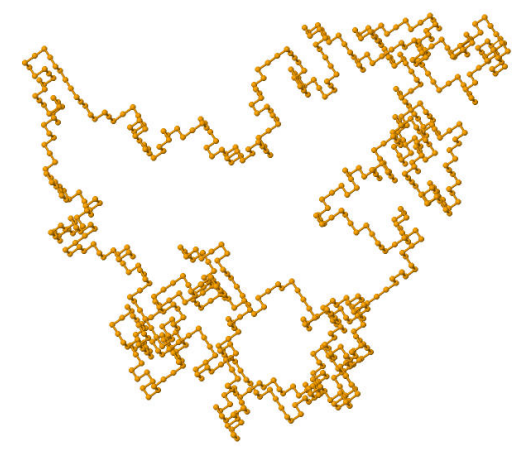

(b)

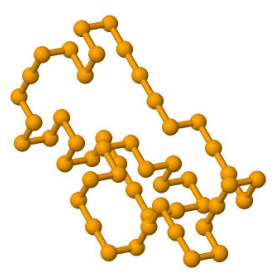

(d)

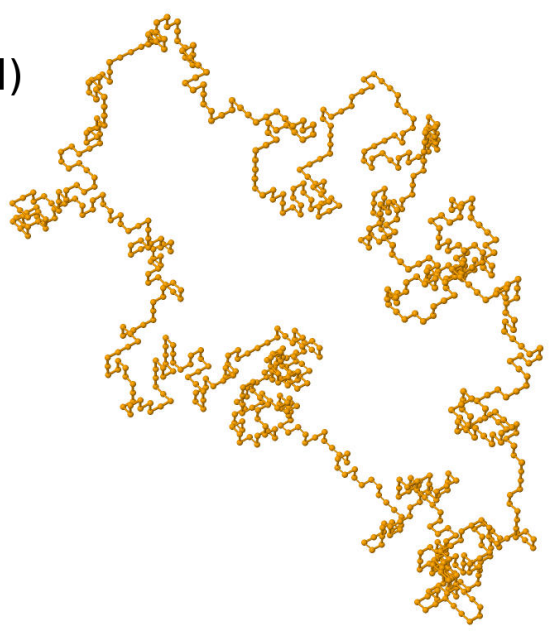

Fig. 6. Typical conformations of chain: $N=50$ and $\rho_{o b s}=0.1$ (a), $N=50$ and $\rho_{\text {obs }}=0.25$ (b), $N=800$ and $\rho_{o b s}=0.1$ (c), $N=800$ and $\rho_{o b s}=0.25(\mathrm{~d})$

the other hand, it contains more locally coiled sequences. The snapshots of a long chain $(N=800)$ at low and high obstacle densities look similar to those of the short chain.

\section{CONCLUSIONS}

In this paper we studied the behavior of cyclic polymer chains in porous media by means of dynamic Monte Carlo simulations. The model chains were built without atomic details as sequences of identical segments whose positions were restricted to vertices of a simple cubic lattice. The presence of small impenetrable obstacles simulated the presence of porous media. The mean size of chains depended on the concentration of obstacles: the longer the chain, the stronger the impact of obstacles. The size of the chain did not change monotonically with the obstacle density, which confirmed that it was a universal behavior when the size of obstacles was comparable with polymer segments. The mobility of the chain decreased with the increase of obstacle density regardless of the polymer length. It was found that the polymer self-diffusion coefficient scaled with the chain length as $N^{b}$ and the value of the exponent $b$ changed from -1 to -1.25 when going from a free chain to porous media with higher obstacle density but still below the percolation threshold.

\section{Acknowledgements}

The computational part of this work was done using the computer cluster at the Computing Center of the Department of Chemistry, University of Warsaw. This work was supported by the Polish Ministry of Science and Higher Education grant N N507 326536.

\section{References}

[1] A. Baumgärtner and M. Muthukumar, A trapped polymer in random porous media, Adv. Chem. Phys. 94, 625-708 (1996).

[2] M. Ediger, Spatially Heterogeneous Dynamics In Supercooled Liquids, Annu. Rev. Phys. Chem. 51, 99-128 (2000).

[3] E. Eisenriegler, Polymers near Surfaces, World Scientific, Singapore 1993.

[4] A. Sikorski, Polymer Chains in Confinement and Porous Media, Solid State Phenom. 138, 451-475 (2008).

[5] G.W. Slater and S.Y. Wu, Reptation, Entropic Trapping, Percolation, and Rouse Dynamics of Polymers in "Random" Environments, Phys. Rev. Lett. 75, 164-167 (1995).

[6] V. Yamakov, D. Stauffer, A. Milchev, G.M. Foo and R.B. Pandey, Crossover Dynamics for Polymer Simulation in Porous Media, Phys. Rev. Lett. 79, 2356-2358 (1997).

[7] V. Yamakov and A. Milchev, Diffusion of a polymer chain in porous media, Phys. Rev. E 55, 1704-1712 (1997). 
[8] G.I. Nixon and W.G. Slater, Relaxation length of a polymer chain in a quenched disordered medium, Phys. Rev. E 60, 3170-3173 (1999).

[9] P.M. Saville and E.M. Sevick, Collision of a Field-Driven Polymer with a Finite-Sized Obstacle: A Brownian Dynamics Simulation, Macromolecules 32, 892-899 (1999).

[10] S.H. Chern and R.D. Coalson, Entropic trapping of a flexible polymer in a fixed network of random obstacles, J. Chem. Phys. 111, 1778-1781 (1999).

[11] A. Dua and B.J. Cherayil, The anomalous diffusion of polymers in random media, J. Chem. Phys. 112, 421-427 (2000).

[12] G.I. Nixon and W.G. Slater, Saturation and entropic trapping of monodisperse polymers in porous media, J. Chem. Phys. 117, 4042-4046 (2002).

[13] A. Bhattacharya, Conformation and drift of a telechelic chain in porous media, J. Phys.: Condens. Matter 16, 52035211 (2004).

[14] A.J. Moreno and W. Kob, Relaxation dynamics of a linear molecule in a random static medium: A scaling analysis, $\mathrm{J}$. Chem. Phys. 121, 380-386 (2004).

[15] G.C. Randall and P.S. Doyle, Collision of a DNA Polymer with a Small Obstacle, Macromolecules 39, 7734-7745 (2006).

[16] R. Chang and A. Yethiraj, Dynamics of Chain Molecules in Disordered Materials, Phys. Rev. Lett. 96, 107802 (2006).

[17] O.A. Hickey and W.G. Slater, The diffusion coefficient of a polymer in an array of obstacles is a non-monotonic function of the degree of disorder in the medium, Phys. Lett. A 364, 448-452 (2007).

[18] A. Balducci and P.S. Doyle, Conformational Preconditioning by Electrophoresis of DNA through a Finite Obstacle Array, Macromolecules 41, 5485-5492 (2008).

[19] B.J. Sung, R. Chang and A. Yethiraj, Swelling of polymers in porous media, J. Chem. Phys. 130, 124908 (2009).

[20] P. Romiszowski, A. Sikorski, Properties of Linear Polymer Chains in Porous Media, J. Non-Crystal. Solids 352, 43034308 (2006).

[21] J.A. Semlyen, Cyclic Polymers (2nd edition), Kluwer, Dordrecht 2000.

[22] T.C.B. McLeish, Polymers Without Beginning or End, Science 297, 2005-2006 (2002).

[23] T.C.B. McLeish, Polymer dynamics: Floored by the rings, Nature Mater. 7, 933-935 (2008).

[24] M. Kapnistos, M. Lang, D. Vlassopoulos, W. PyckhoutHintzen, D. Richter, D. Cho, T. Chang and M. Rubinstein, Unexpected power-law stress relaxation of entangled ring polymers, Nature Mater. 7, 997-1002 (2008).

[25] C.W. Bielawski, D. Benitez and R.H. Grubbs, An "Endless" Route to Cyclic Polymers, Science 297, 2041-2044 (2002).

[26] G. Beaucage, A.S. Kulkarni, Dimensional Description of Cyclic Macromolecules, Macromolecules 43, 532-537 (2010).

[27] V. Arrighi, S. Gagliardi, A.C. Dagger, J.A. Semlyen, J.S. Higgins and M.J. Shenton, Conformation of Cyclics and Linear Chain Polymers in Bulk by SANS, Macromolecules 37, 8057-8065 (2004).

[28] R.M. Robertson and D.E. Smith, Strong effects of molecular topology on diffusion of entangled DNA molecules, Proc. Natl. Acad. Sci. U.S.A. 104, 4824-4827 (2007).

[29] F. Baldelli Bombelli, F. Gambinossi, M. Lagi, D. Berti, G. Caminati, T. Brown, F. Sciortino, B. Norden and P. Baglioni, DNA Closed Nanostructures: A Structural and Monte Carlo Simulation Study, J. Chem. Phys. B 112, 15283-15294 (2008).
[30] S.P. Obukhov, M. Rubinstein and T. Duke, Dynamics of a Ring Polymer in a Gel, Phys. Rev. Lett. 73, 1263-1266 (1994).

[31] J. Klein, Dynamics of entangled linear, branched, and cyclic polymers, Macromolecules 19, 105-118 (1986).

[32] G. Zifferer and W. Preusser, Monte Carlo Simulation Studies of the Size and Shape of Ring Polymers, Macromol. Theory Simul. 10, 397-407 (2001).

[33] J. Reiter, Monte Carlo simulations of linear and cyclic chains on cubic and quadratic lattices, Macromolecules 23, 3811-3816 (1990).

[34] M. Bishop and J.P.J. Michels, The shape of ring polymers, J. Chem. Phys. 82, 1059-1061 (1985).

[35] M. Bishop, J.P.J. Michels, Scaling in three-dimensional linear and ring polymers, J. Chem. Phys. 84, 444-446 (1986).

[36] M. Bishop and C.J. Saltiel, Polymer shapes in two, four, and five dimensions, J. Chem. Phys. 88, 3976-3982 (1985).

[37] J. Suzuki, A. Takano and Y. Matsushita, Topological effect in ring polymers investigated with Monte Carlo simulation, J. Chem. Phys. 129, 034903 (2008).

[38] S. Brown and G. Szamel, Structure and dynamics of ring polymers, J. Chem. Phys. 108, 4705-4708 (1998).

[39] S. Brown and G. Szamel, Computer simulation study of the structure and dynamics of ring polymers, J. Chem. Phys. 109, 6184-6192 (1998).

[40] N. Kanaeda and T. Deguchi, Diffusion of a ring polymer in good solution via the Brownian dynamics with no bond crossing, J. Phys. A: Math. Theor. 41, 145004 (2008).

[41] S. Brown, T. Lenczycki and G. Szamel, Influence of topological constraints on the statics and dynamics of ring polymers, Phys. Rev. E 63, 052801 (2001).

[42] A. Vettorel, A.Y. Grossberg and K. Kremer, Statistics of polymer rings in the melt: a numerical simulation study, Phys. Biol. 6, 025013 (2009).

[43] G. Tsolou, N. Stratikis, C. Baig, P.S. Stephanou and V.G. Mavrantzas, Melt Structure and Dynamics of Unentangled Polyethylene Rings: Rouse Theory, Atomistic Molecular Dynamics Simulation, and Comparison with the Linear Analogues, Macromolecules 43, 10692-10713 (2010).

[44] J. Suzuki, A. Takano, T. Deguchi and Y. Matsushita, Dimension of ring polymers in bulk studied by Monte-Carlo simulation and self-consistent theory, J. Chem. Phys. 131, 144902 (2009).

[45] T. Pakula and K. Jeszka, Simulation of Single Complex Macromolecules. 1. Structure and Dynamics of Catenanes, Macromolecules 32, 6821-6830 (1999).

[46] J. Reiter, Monte Carlo study of diffusion of an ideal ring polymer in a network of obstacles on a cubic and a square lattice, J. Chem. Phys. 95, 1290-1294 (1991).

[47] D. Gersappe and M. Olvera de la Cruz, A Monte Carlo Study of Ring Polymers in Disordered Systems, Mol. Simulat. 13, 267-283 (1994).

[48] B.V.S. Iyer, A.K. Lele, V.A. Juvekar and R.A. Mashelkar, Self-Similar Dynamics of a Flexible Ring Polymer in a Fixed Obstacle Environment: A Coarse-Grained Molecular Model, Ind. Eng. Chem. Res. 48, 9514-9522 (2009).

[49] J. Skolnick and A. Kolinski, Dynamics of Dense Polymer Systems: Computer Simulations and Analytic Theories, Adv. Chem. Phys. 77, 223-278 (1990).

[50] A. Koliński, M. Vieth i A. Sikorski, Collapse of Semiflexible Polymers in Two Dimensions. Monte Carlo Simulations, Acta Phys. Polon. A 79, 601-612 (1991).

[51] K. Binder, M. Müller, J. Baschnagel, Polymer Models on the Lattice, in: M. Kotelyanskii, D.N. Theodorou (eds.) Simulation Methods for Polymers, Marcel Dekker, NewYorkBasel, p. 125-146, 2004. 
[52] A. Takano, Y. Ohta, K. Masuoka, K. Matsubara, T. Nakano, A. Hieno, M. Itakura, K. Takahashi, S. Kinugasa, D. Kawaguchi, Y. Takahashi, Y. Matsushita, Radii of gyration of ring-shaped polystyrenes with high purity in dilute solutions, Macromolecules 45, 369-373 (2012).

[53] A.Y. Grosberg, Critical Exponents for Random Knots, Phys. Rev. Lett. 85, 3858-3861 (2000).
[54] A. Dobay, J. Dubochet, K. Millett, P.-E. Sottas, A. Stasiak, Scaling behavior of random knots, Proc. Natl. Acad. Sci. U.S.A. 100, 5611-5615 (2003).

[55] A. Kolinski, J. Skolnick, R. Yaris, Monte Carlo studies on the long time dynamic properties of dense cubic lattice multichain systems. I. The homopolymeric melt, J. Chem. Phys. 86, 7164-7173 (1987).

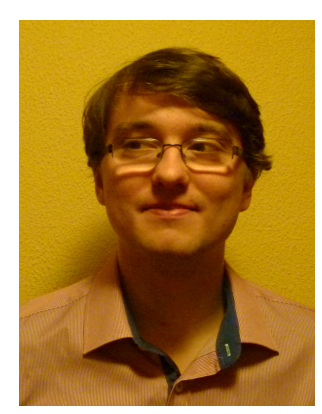

Aleksander Kuriata received his Bachelor's degree in Chemistry from the University of Warsaw in 2013. Currently he is an MSc student at the Laboratory of Theory of Biopolymers at the University of Warsaw. He is interested in polymer physics.

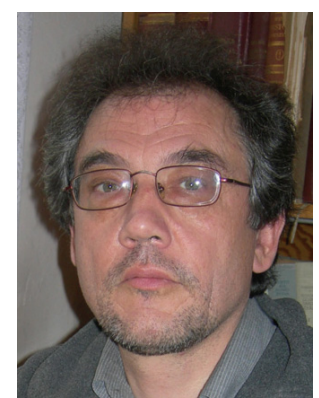

Andrzej Sikorski graduated from the Department of Chemistry, University of Warsaw in 1980. He completed his PhD Thesis in Chemistry in 1986 in the same Department. In 1987/89 and 1990/91 he worked as a postdoc and visiting professor in Washington University in St. Louis and in The Scripps Research Institute in La Jolla. In 1995 he obtained DSc degree in Chemistry. His main scientific interest is the theory and simulation of polymers at interfaces and in confined geometries. 